\title{
Herpes simplex virus type-2 stimulates HIV-1 replication in cervical tissues: implications for HIV-1 transmission and efficacy of anti-HIV-1 microbicides
}

\author{
C Rollenhagen ${ }^{1,2}$, MJ Lathrop ${ }^{2}$, SL Macura ${ }^{1}$, GF Doncel ${ }^{3}$ and SN Asin ${ }^{1,2}$
}

Herpes Simplex virus Type-2 (HSV-2) increases the risk of HIV-1 acquisition, yet the mechanism for this viral pathogen to regulate the susceptibility of the cervicovaginal mucosa to HIV-1 is virtually unknown. Using ex vivo human ectocervical tissue models, we report greater levels of HIV-1 reverse transcription, DNA integration, RNA expression, and virions release in HIV-1/HSV-2 co-infected tissues compared with HIV-1 only infected tissues $(P<0.05)$. Enhanced HIV-1 replication was associated with increased CD4, CCR5, and CD38 transcription $(P<0.05)$ and increased number of $\mathrm{CD}^{+} /{ }^{\mathrm{CCR}} 5^{+} / \mathrm{CD} 38^{+}$T cells in HIV-1/HSV-2 co-infected tissues compared with tissues infected with HIV-1 alone. Tenofovir (TFV) $1 \%$ gel, the leading microbicide candidate, demonstrated only partial protection against HIV-1, when applied vaginally before and after sexual intercourse. It is possible that mucosal inflammation, in particular that induced by HSV-2 infection, may have decreased TFV efficacy. HSV-2 upregulated the number of HIV-1-infected cells and elevated the concentration of TFV needed to decrease HIV-1 infection. Similarly, only high concentrations of TFV inhibited HSV-2 replication in HIV-1/HSV-2-infected tissues. Thus, HSV-2 co-infection and mucosal immune cell activation should be taken into consideration when designing preventative strategies for sexual transmission of HIV-1.

\section{INTRODUCTION}

Epidemiological studies suggest that HSV-2 increases the risk of HIV-1 acquisition. ${ }^{1-3}$ In populations with HSV-2 prevalence of $80 \%$, nearly $50 \%$ of HIV-1 infections are attributable to an HSV-2 co-infection. ${ }^{4}$ Several mechanisms for this increased susceptibility have been proposed, including breaches of the epithelial barrier that facilitate HIV-1 access to CD4 ${ }^{+}$T lymphocytes $^{5}$ and persistence of CD4 ${ }^{+}$T-cell infiltrates susceptible to HIV-1 beyond HSV-2 lesion healing. ${ }^{6} \mathrm{HSV}-2$ may also alter innate mucosal immunity and induce an inflammatory response. ${ }^{7-11}$ By using ex vivo cervical cultures, Horbul et al. ${ }^{5}$ demonstrated that HSV-1 or HSV-2 induces multinuclear giant cell formation and widespread epithelial cell damage. Subsequent tissue exposure to HIV-1 revealed co-localization of HSV and HIV antigens in single cells. This study, however, did not quantify nor characterize the phenotype of the co-infected cells. Similarly, analysis of cellular infiltrates from biopsies of HSV-2 lesions demonstrated $\mathrm{CD} 4{ }^{+} \mathrm{CCR}^{+}{ }^{+} \mathrm{T}$ cells harboring greater concentrations of integrated HIV-1 DNA compared with control samples not affected by HSV-2. ${ }^{6} \mathrm{CD} 4^{+}$T-cell infiltration, however, was evaluated in the context of HSV-2 reactivation and in biopsies from genital skin rather than mucosal surfaces of female reproductive tract (FRT) sites. Using a novel nonpolarized human ectocervical tissue model of ex vivo viral infection, ${ }^{12-14}$ we evaluated the impact of HSV-2 on HIV-1 infection and replication. We focused on tissues from lower FRT sites such as ectocervix, as they are likely the first to encounter these viruses.

Mucosal HSV-2 reactivation and primary infection are associated with sequential infiltration of neutrophils, natural

\footnotetext{
${ }^{1}$ V.A. Medical Center, White River Junction, Vermont, USA. ${ }^{2}$ Department of Microbiology and Immunology, Dartmouth Medical School, Lebanon, New Hampshire, USA and ${ }^{3}$ CONRAD, Eastern Virginia Medical School, Norfolk, Virginia, USA. Correspondence: SN Asin (Susana.Asin@Dartmouth.EDU)

These results were presented at the Keystone Symposia, "Immune Activation in HIV infection: Basic Mechanisms and Clinical Implications". April 3-8. 2013. Breckenridge, CO, USA
}

Received 19 September 2013; accepted 3 January 2014; published online 5 February 2014. doi:10.1038/mi.2014.3 
killer cells, macrophages, and CD4 ${ }^{+} \mathrm{T}$ lymphocytes. ${ }^{15-19}$ As the ability of $\mathrm{CD} 4^{+}$cells to support HIV-1 replication depends on HIV-1 co-receptors, differentiation and activation marker expression, ${ }^{20-22}$ the presence of activated $\mathrm{CD} 4{ }^{+} \mathrm{T}$ cells and macrophages within herpetic lesions creates an ideal microenvironment for HIV-1 infection. Moreover, the release of proinflammatory cytokines or chemokines from HSV-2-infected cells or HSV-2-antigen-stimulated $\mathrm{CD}^{+}{ }^{+} \mathrm{T}$ cells may indirectly support HIV-1 replication. ${ }^{23,24}$ In HSV-2-infected women, pro-inflammatory factors can induce activation and proliferation of mucosal HIV-1 target cells or stimulate signaling pathways leading to enhanced HIV-1 transcription within $\mathrm{CD} 4{ }^{+}$T cells harboring HIV-1 integrated DNA. Thus, immune cell activation by HSV-2 is likely an additional mechanism to enhance HIV-1 infection.

We report greater levels of HIV-1 replication in HIV-1/HSV-2infected ectocervical tissues compared with tissues infected with HIV-1 alone. HSV-2 increased HIV-1 reverse transcription, DNA integration, viral RNA expression, and p24 antigen levels $(P<0.05)$. Characterization of the molecular mechanism revealed earlier kinetics of CD4, CCR5, and CD38 upregulation in HIV-1/HSV-2-infected tissues compared with tissues infected with either virus alone $(P<0.05)$, suggesting generalized mucosal activation.

Given HSV-2 infection rates greater than $60 \%$ in women at risk for HIV-1, ${ }^{25}$ it is relevant to define the impact of this viral pathogen on the anti-HIV potency of microbicides, i.e., vaginal formulations aimed at preventing HIV-1 sexual transmission. Tenofovir (TFV) $1 \%$ gel, the leading candidate, demonstrated overall $39 \%$ protection and $54 \%$ in the most adherent women, when applied vaginally before and after sexual intercourse. ${ }^{26}$ In our experiments, HSV-2 upregulated the frequency of HIV-1infected cells, to the extent that, at low concentrations, TFV decreased HIV-1 replication in tissues infected with HIV-1 alone but was not effective in HIV-1/HSV-2-infected tissues. Similarly, low concentrations of TFV did not prevent HSV-2 replication in dually infected tissues.

HSV-2 enhances ectocervical tissue susceptibility to HIV-1 and decreases the anti-HIV potency of TFV. Mucosal immune cell activation by HSV-2 increases the frequency of activated/ infected $\mathrm{CD}^{+}{ }^{+}$cells and elevates the concentration of TFV needed to prevent genital HIV-1 infection.

\section{RESULTS}

HSV-2 infection enhances HIV-1 replication in ectocervical tissues

In initial experiments, ectocervical tissue blocks, established as previously described, ${ }^{12-14}$ were infected with HSV-2 at $4 \times 10^{4}$ plaque forming units (p.f.u.) per $\mathrm{ml}$ of tissue culture supernatant. After $6 \mathrm{~h}$ of incubation at $37^{\circ} \mathrm{C}$, tissues were exposed to $1 \times 10^{4} 50 \%$-tissue culture infective dose (TCID 50) per ml of

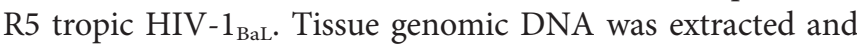
evaluated for HIV-1 reverse transcription and integration by real-time (RT)-PCR on day 11 after infection. Complete HIV-1 reverse transcription was used to confirm productive HIV-1 infection. As lymph node CD4 ${ }^{+} \mathrm{T}$ cells from HIV-1-infected individuals each contains one copy of integrated viral DNA, ${ }^{27}$ HIV-1 integration was used as a surrogate of number of infected cells. We detected a threefold increase in levels of fully reverse transcribed and integrated HIV-1 DNA in HIV-1/HSV-2infected tissues compared with tissues infected with HIV-1 alone, indicating greater number of HIV-1-infected cells in dually infected tissues (Figure 1a and $\mathbf{b}$ ). The ratio of reverse transcribed to integrated HIV-1 DNA was nearly the same, suggesting that the majority of reverse transcripts integrate into the host cell genome. To test whether the HIV-1-infected cells were producing virus, we evaluated expression of the HIV-1 early transcripts tat and rev in total RNA isolated from tissues infected with either HIV-1 alone or in combination with HSV-2 at the same titers proven to upregulate HIV-1 infection. Results from RT-PCR assays indicated greater levels of HIV-1 transcripts, a threefold increase, in HIV-1/HSV-2-infected tissues compared with tissues infected with HIV-1 alone on day 6 after infection (Figure 1c), suggesting enhanced expression of newly transcribed HIV-1 genes in co-infected tissues. Completion of HIV-1 transcription might not result in a fully productive infection; hence, we evaluated whether greater levels of HIV-1 DNA and RNA expression were associated with increased viral release. Therefore, HIV-1 p24 antigen levels were measured by using ELISA on days 11 and 21 after infection in the culture supernatants of tissues that demonstrated enhanced HIV-1 DNA and RNA expressions. We found greater HIV-1 p24 levels in HIV-1/HSV-2-infected tissues (Figure 1d, grey line) compared with tissues infected with HIV-1 alone through day 21 (Figure 1d, black line). To test whether upregulation of HIV-1 replication was associated with productive Herpes infection, HSV-2 DNA expression was used as a surrogate of viral replication and evaluated in culture supernatants of co-infected tissues by quantitative RT-PCR. Compared with day 0, HIV-1/HSV-2infected tissues had increased HSV-2 DNA levels by 14- and 9-fold on days 4 and 11 after infection, respectively (Figure 1d). Thus, HSV-2 enhances the frequency of HIV-1-infected cells producing virus in ectocervical tissues.

\section{HSV-2 enhances, whereas HIV-1 decreases CD4 and CCR5 RNA expression in ectocervical tissues}

Our findings of enhanced HIV-1 integration and viral p24 release in co-infected tissues suggest greater frequency of $\mathrm{CD}^{+}{ }^{+}$cells replicating virus under settings of HSV-2 coinfection. As cervicovaginal tissues support productive HIV-1 infection in activated $\mathrm{CD} 4{ }^{+} \mathrm{CCR}^{+}{ }^{+} \mathrm{T}$ cells, ${ }^{28}$ we postulated that the early inflammatory response to HSV-2 creates an ideal microenvironment for HIV-1 infection providing the virus with additional target cells. Thus, we evaluated transcription levels of the HIV-1 receptor CD4 and co-receptor CCR5 in singly and dually infected tissues. Tissues infected with HIV-1 or HSV-2 alone served as controls, establishing the contribution of either virus to receptor expression. Values of receptor expression were expressed relative to baseline defined in uninfected tissues (set to 1).

On day 7, tissues infected with HSV-2 or HSV-2 and HIV-1 had increased levels of CD4 RNA expression by 12.3-fold and 

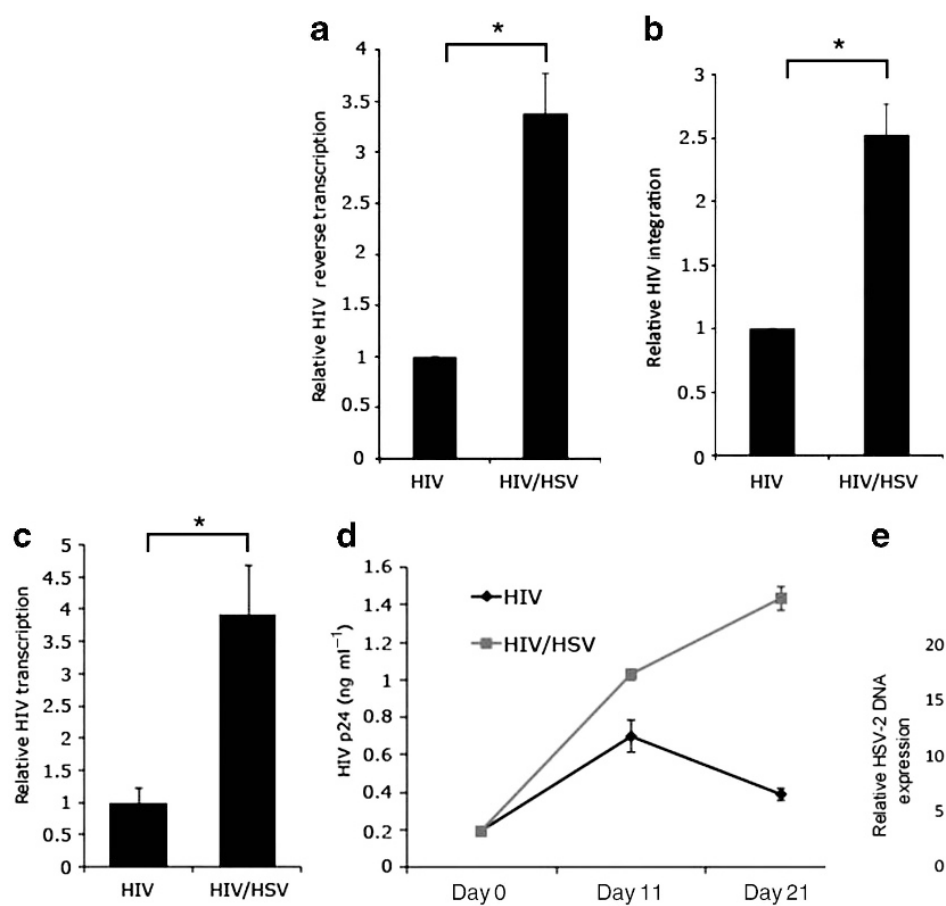

e

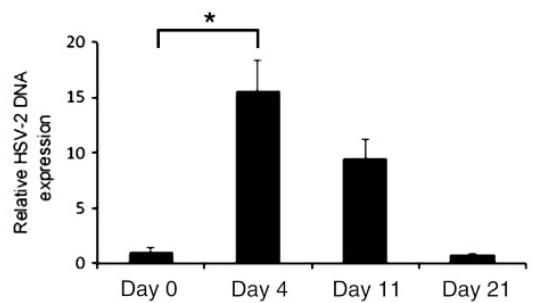

Figure 1 HSV-2 enhances HIV-1 replication in ectocervical tissues. Levels of complete reverse transcribed (a) and integrated (b) HIV-1 DNA in HIV-1and HIV-1/HSV-2-infected ectocervical tissues were quantified by RT-PCR on day 11 after infection. Data were normalized to human $\beta$-actin. Levels of HIV-1 transcription (c) in HIV-1- and HIV-1/HSV-2-infected ectocervical tissues were quantified by RT-PCR on day 6 after infection. Data were normalized to glyceraldehyde 3 phosphate dehydrogenase (GAPDH). HIV-1 p24 levels (d) (ng ml ${ }^{-1}$ ) in HIV-1-and HIV-1/HSV-2-infected ectocervical tissues were measured after washing residual input virus (day 0) and again on days 11 and 21 after infection. Results were consistent among donor-matched samples from six donors and are shown as the mean $\pm \mathrm{s}$.d. from three replicates from one representative experiment with each condition tested in triplicate. When data from all six donors were averaged, we detected a 5.8-fold increase in reverse transcribed and a 0.5-fold increase in integrated HIV-1 DNA in HIV-1/ HSV-2-infected tissues compared with tissues infected with HIV-1 alone. Similarly, we found a 4.5-fold increase in HIV-1 transcription, and, on day 11, the earliest time point where newly produced virus was released, we found a 0.7 -fold increase in $p 24$ levels. This increase was greater at day 21 . ${ }^{*} P<0.05$ for HIV-1 vs. HIV-1/HSV-2-infected tissues. (e) HSV-2 DNA levels in the culture supernatant of HIV-1/HSV-2-infected ectocervical were measured by RTPCR on days 4, 11, and 21 after infection. All data were normalized to day 0 . Results were consistent among donor-matched samples from three donors and are shown as the mean \pm s.d. of one representative experiment with each condition tested in triplicate. ${ }^{*} P<0.05$ for HIV-1/HSV-2-infected tissues day 0 vs. days 4 and 11 after infection.

6.8-fold, respectively, compared with HIV-1-infected tissues (Figure 2a). Susceptibility of $\mathrm{CD} 4^{+}$cells to HIV-1 depends on their pattern of HIV-1 co-receptor expression. ${ }^{29}$ HIV-1 variants that infect the cervicovaginal mucosa primarily use CCR5. ${ }^{30-32}$ We therefore evaluated CCR5 transcription levels in tissues from the same experiment. We detected a twofold increase in CCR5 expression in tissues infected with either HSV-2 alone or a combination of HSV-2 and HIV-1 compared with those infected with HIV-1 alone (Figure 2b). Thus, by enhancing CD4 and CCR5 expressions, HSV-2 likely provides additional target cells that are infected and depleted by HIV-1. Furthermore, HIV-1 seems to deplete cells of CD4 ${ }^{+}$CCR5 ${ }^{+}$ phenotype in both co-infected and singly infected tissues. To test this hypothesis, we evaluated HIV-1 receptor/co-receptor expression at earlier time points after infection. On day 3 , ectocervical tissues infected with HIV-1 alone or a combination of HIV-1 and HSV-2 had enhanced levels of CD4 transcription compared with baseline (Figure 3a). On day 5, CD4 levels were further increased in tissues infected with HIV-1 or HSV-2 alone. Conversely, CD4 RNA expression on day 5 was significantly downregulated in HIV-1/HSV-2-infected tissues compared with day 3 levels (Figure 3a). On day 5, tissues infected with HSV-2 or HIV-1 alone had enhanced levels of CCR5 transcription. The increase was about threefold compared with that on day 3 (Figure 3b). Conversely, levels of CCR5 RNA expression were downregulated in HIV-1/HSV-2-infected ectocervical tissues in comparison with that on day 3 (Figure 3b).

\section{CD38 RNA expression is decreased in HIV-1/HSV-2-infected ectocervical tissues}

HIV-1 replication is more efficient in activated compared with resting $\mathrm{CD}^{+}{ }^{+} \mathrm{T}_{\text {cells. }}{ }^{20-22}$ As $\mathrm{CD} 38$ expression defines a subpopulation of activated $\mathrm{CD} 4^{+} \mathrm{T}$ cells that is preferentially infected by HIV-1 in cervicovaginal tissues ex vivo, ${ }^{28}$ we evaluated ectocervical tissues from the same experiment for CD38 transcription on both days 3 and 5 after infection.

On day 3, we detected 10-fold higher levels of CD38 RNA expression in HIV-1/HSV-2-infected tissues compared with tissues infected with HSV-2 or HIV-1 alone (Figure 4, black bars). On day 5 , compared with that on day 3 , levels of $\mathrm{CD} 38$ RNA expression were 10-fold higher in tissues infected with either virus alone. Conversely, CD38 levels decreased on day 5 by threefold in HIV-1/HSV-2-infected tissues (Figure 4). 

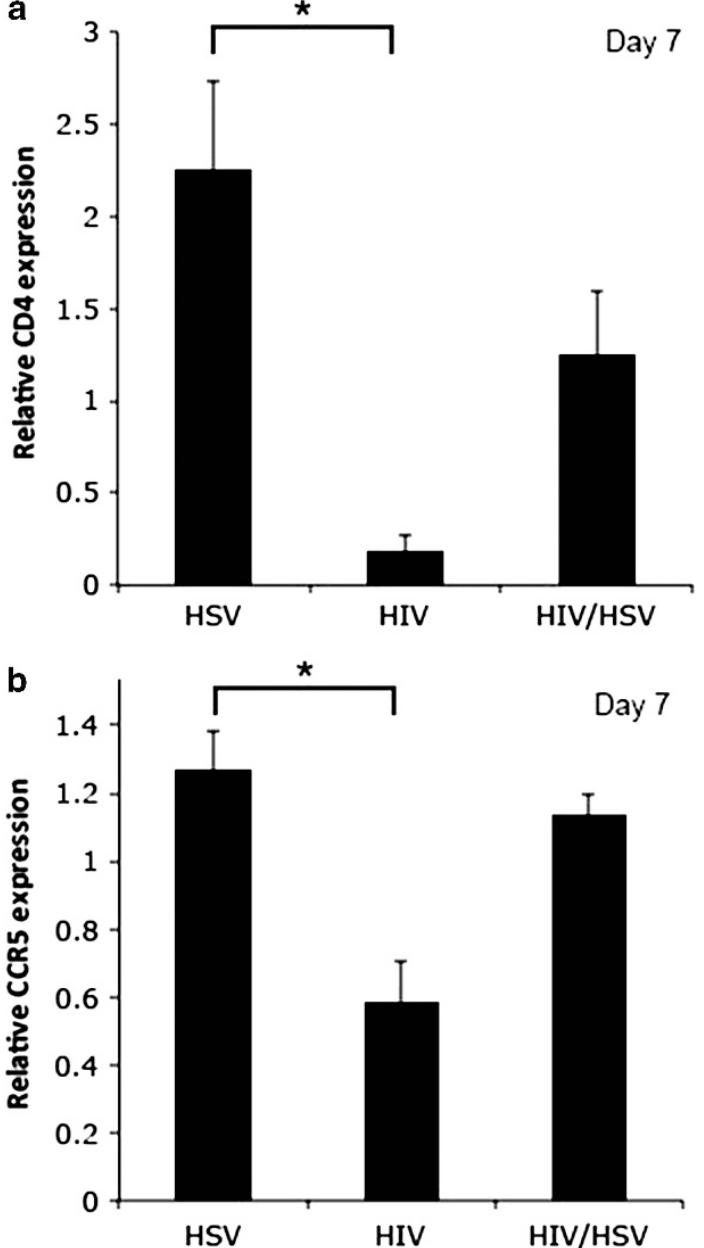

Figure 2 Enhanced CD4 and CCR5 expression in HIV-1/HSV-2- and HSV-2-infected ectocervical tissues at day 7 of infection. Levels of CD4 (a) and CCR5 (b) transcription in HSV-2, HIV-1, and HIV-1/HSV-2-infected ectocervical tissues were quantified by RT-PCR on day 7 after infection. All data were normalized to GAPDH. Results were consistent among donormatched samples from three donors and are shown as the mean \pm s.d. of one representative experiment with each condition tested in triplicate. ${ }^{\star} P<0.05$ for HIV-1- vs. HSV-2- and HIV-1/HSV-2-infected tissues.

Our findings indicate slower kinetics of CD38 induction in HIV -1 and HSV-2 singly compared with dually infected tissues. As CD38-activated $\mathrm{CD} 4^{+} \mathrm{T}$ cells are preferentially infected and depleted by HIV-1 in the cervicovaginal mucosa, ${ }^{28}$ our results suggest that induction of CD38 expression on HIV-1 target cells by either virus alone or in combination render them susceptible to infection and depletion by HIV-1. This pattern of CD4, CCR5, and CD38 expression was associated with enhanced HIV-1 infection and replication in HIV-1/HSV-2-infected tissues compared with tissues infected with HIV-1 alone (Figure 1a-d).

Tenofovir restores CD38 RNA expression to baseline levels Our findings thus far suggest that HIV-1 or HSV-2 enhances CD38 expression on HIV-1 target cells. Furthermore, HIV-1 seems to preferentially deplete target cells of activated CD $38^{+}$
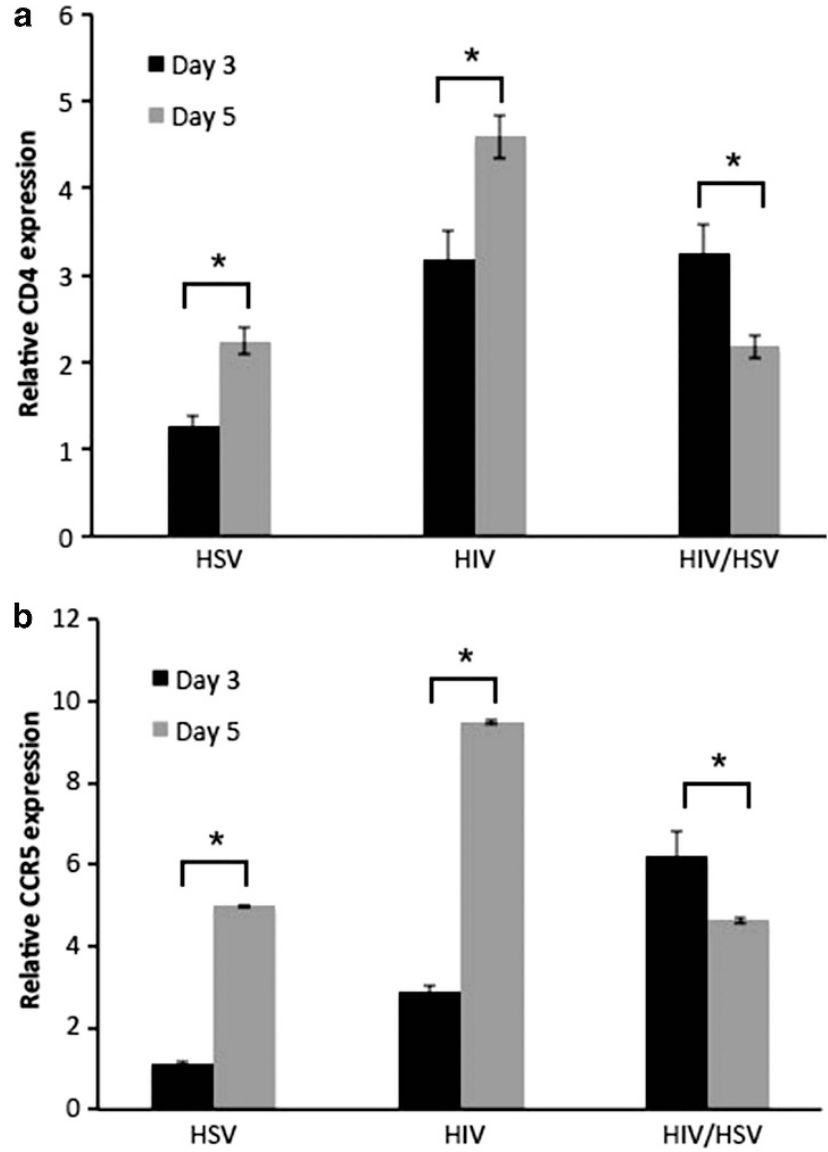

Figure 3 Decreased CD4 and CCR5 expression in HIV-1/HSV-2infected ectocervical tissues at day 5 of infection. Levels of CD4 (a) and CCR5 (b) transcription in HSV-2-, HIV-1-, and HIV-1/HSV-2-infected ectocervical tissues were quantified by RT-PCR on days 3 (black bars) and 5 (grey bars) after infection. All data were normalized to GAPDH. Results were consistent among donor-matched samples from three donors and are shown as the mean \pm s.d. of one representative experiment with each condition tested in triplicate. ${ }^{*} P<0.05$ for days 3 vs. 5 HSV-2-, HIV-1- and HIV-1/HSV-2-infected tissues.

phenotype. Thus, we evaluated CD38 expression in donormatched ectocervical tissues infected with HSV-2 or HIV-1 alone or in combination, and left untreated or treated with TFV. On day 7, TFV at $50 \mu \mathrm{g} \mathrm{ml}^{-1}$, a concentration demonstrated to inhibit HIV-1 reverse transcriptase and HSV-2 DNA polymerase in ectocervical tissues, ${ }^{33,34}$ restored CD38 expression in HIV-1, HSV-2, and HIV-1/HSV-2-infected tissues to baseline levels in uninfected TFV-treated control tissues (Figure 5a, compare black and grey bars). Furthermore, TFV did not significantly alter baseline levels of CD38 expression in uninfected tissues. Consistent with our day 5 findings (Figure 4), on day 7, levels of CD38 RNA expression were twofold higher in HSV-2-infected tissues, whereas CD38 expression decreased by eightfold in HIV-1/HSV-2-infected tissues compared with baseline (Figure 5a, black bars). In addition, on day 7, levels of CD38 expression in HIV-1-infected tissues were similar to those in uninfected control tissues. As, on day $5, \mathrm{CD} 38$ expression levels were 12 -fold higher than baseline 


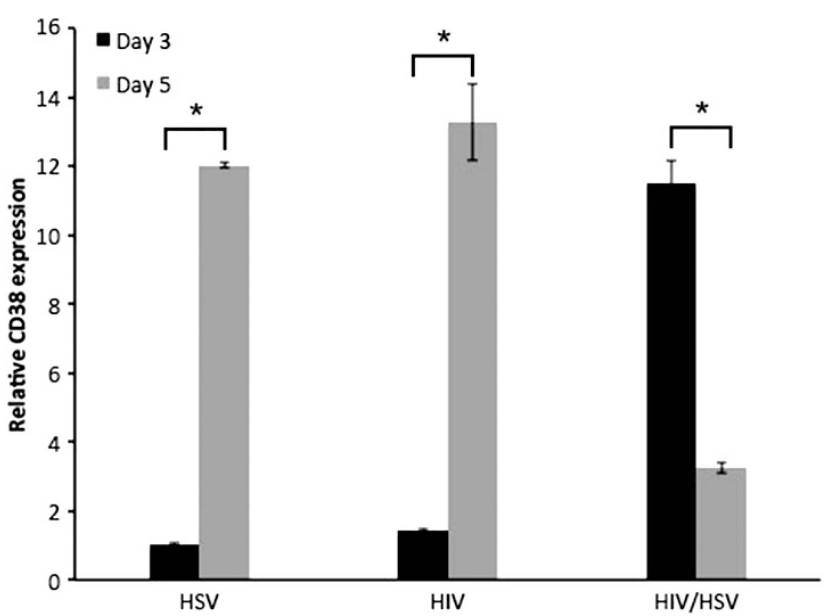

Figure 4 Decreased CD38 expression in HIV-1/HSV-2-infected ectocervical tissues. Levels of CD38 transcription in HSV-2, HIV-1, and HIV-1/HSV-2-infected ectocervical tissues were quantified by RT-PCR on days 3 (black bars) and 5 (grey bars) after infection. All data were normalized to GAPDH. Results were consistent among donor-matched samples from three donors and are shown as the mean \pm s.d. of one representative experiment with each condition tested in triplicate. ${ }^{\star} P<0.05$ for days 3 vs. 5 HSV-2-, HIV-1- and HIV-1/HSV-2-infected tissues.

in HIV-1-infected tissues, our findings indicate downregulation of CD38 expression by HIV-1 on day 7 of infection. To test if modulation of CD38 expression was due to inhibition of productive viral infection, we evaluated supernatants from the same experiment for HIV-1 p24 levels and HSV-2 DNA expression. On day 11, TFV decreased HIV-1 p24 levels, a 5.6-fold and an 8.6-fold reduction in HIV-1- and HIV-1/ HSV-2-infected tissues, respectively (Figure 5b). Similarly, on day 7 , TFV decreased HSV-2 replication by 1.9 -fold and 18.6-fold in HSV-2- and HIV-1/HSV-2-infected tissues, respectively (Figure 5c). In comparing results between singly and dually infected tissues, we observed 1.5-fold higher levels of HIV-1 p24 and 40-fold higher levels of HSV-2 DNA in HIV-1/ HSV-2-infected tissues compared with tissues infected with either virus alone, indicating a synergistic stimulatory interaction between these viruses. Overall, these findings suggest that productive infection by HIV-1 or HSV-2 induces CD38 expression in ectocervical tissues. Furthermore, this induction requires HIV-1 or HSV-2 replication, as the replication-inhibiting antiviral TFV restores CD38 expression to baseline.

HSV-2 decreases the anti-HIV-1 activity of low doses of TFV Given the ability of HSV-2 to enhance HIV-1 infection and replication in the genital mucosa, and the likelihood that women receiving TFV 1\% gel, the leading microbicide candidate in clinical effectiveness trials, have tissue drug concentrations below TFV's in vitro effective (anti-HSV-2) concentrations (around $150 \mu \mathrm{g} \mathrm{g}^{-1}$ ), ${ }^{33}$ we evaluated the impact of HSV-2 on the anti-HIV-1 potency of this compound. We selected drug concentrations within and below the range of 200 to $10 \mu \mathrm{g} \mathrm{ml}^{-1}$; the compound concentrations present in cervicovaginal
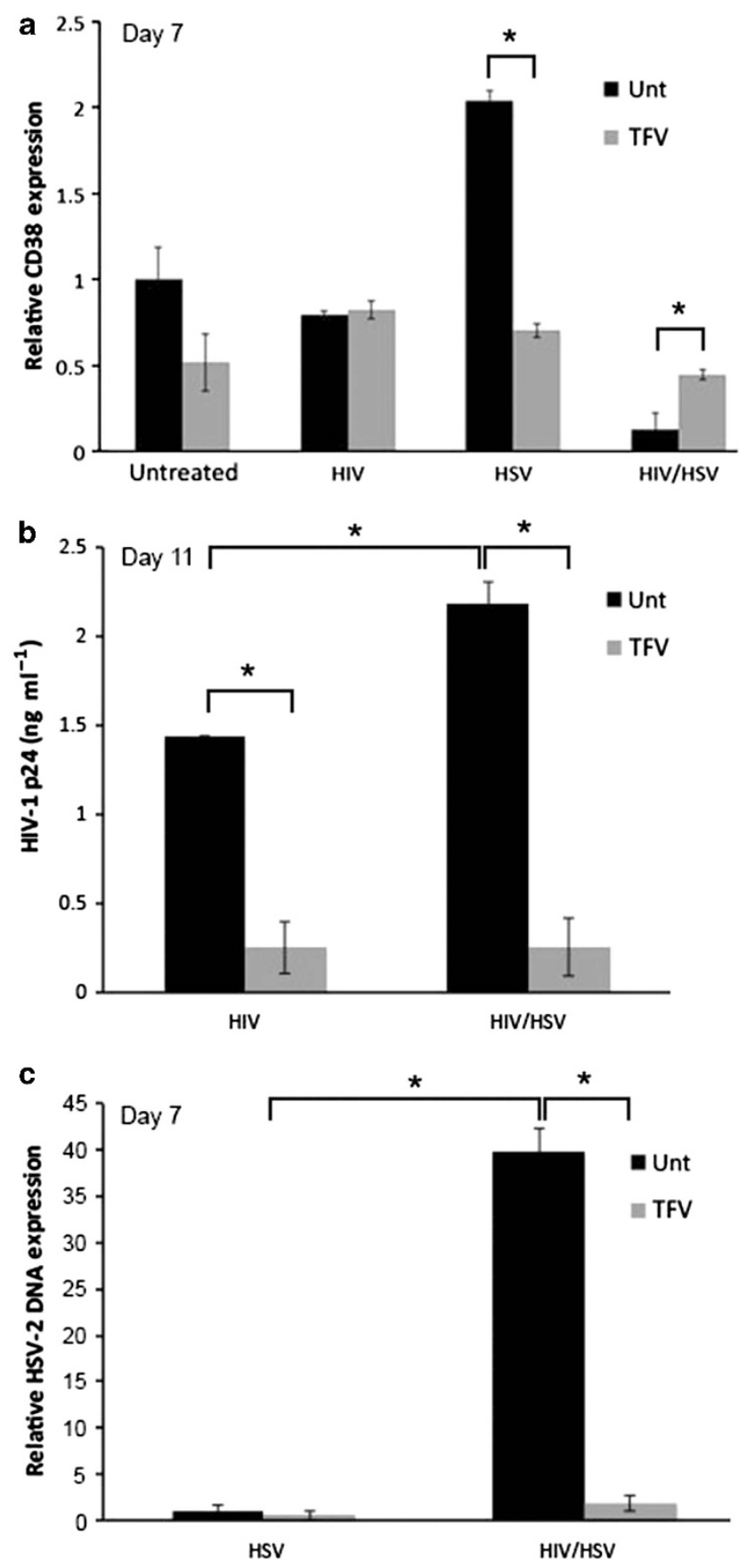

Figure 5 TFV restores CD38 expression to baseline levels in HSV-2-, HIV-1- and HIV-1/HSV-2-infected ectocervical tissues ex vivo. (a) Levels of CD38 transcription in uninfected, HIV-1-, HSV-2- and HIV-1/HSV-2infected ectocervical tissues left untreated (black bars) or treated with TFV (grey bars) at $50 \mathrm{\mu g} \mathrm{ml}^{-1}$ were quantified by RT-PCR on day 7 after infection, one of the earliest time point where TFV displayed anti-viral activity. All data were normalized to GAPDH. (b) HIV-1 p24 levels (ng ml ${ }^{-1}$ ) in HIV-1- and HIV-1/HSV-2-infected ectocervical tissues left untreated (black bars) or treated with TFV at $50 \mu \mathrm{g} \mathrm{ml}^{-1}$ (grey bars) were measured by ELISA on day 11 after infection. (c) HSV-2 DNA levels in the culture supernatant of HSV-2- and HIV-1/HSV-2-infected ectocervical tissues left untreated (black bars) or treated with TFV at $50 \mu \mathrm{g} \mathrm{ml}^{-1}$ (grey bars) were measured by RT-PCR on day 7 after infection. All data were normalized to day 0 . Results were consistent among donor-matched samples from three donors and are shown as the mean \pm s.d. of one representative experiment with each condition tested in triplicate. ${ }^{\star} P<0.05$ for TFV-treated vs. untreated HVS-2-, HIV-1- and HIV-1/HSV-2infected tissues. 
biopsies following vaginal application of TFV $1 \%$ gel. ${ }^{25}$ In these experiments, ectocervical tissues were infected with HSV-2, treated with TFV at $100 \mu \mathrm{g} \mathrm{ml}^{-1}$ or $1 \mu \mathrm{g} \mathrm{ml}^{-1}$ for additional $6 \mathrm{~h}$, and infected with HIV-1. The next day, tissues were washed and further cultured for additional 21 days. HIV-1 p24 levels were measured in the culture supernatants through day 21 by ELISA. Controls included TFV-treated tissues infected only with HIV-1 and tissues infected with HIV-1 alone or in combination with HSV-2 in the absence of TFV. TFV at $100 \mu \mathrm{g} \mathrm{ml}^{-1}$ inhibited HIV-1 replication in HIV-1 (Figure 6a, light gray line triangle) and HIV-1/HSV-2-infected tissues (Figure 6a, light grey line square). In contrast, TFV at $1 \mu \mathrm{g} \mathrm{ml}^{-1}$ decreased HIV-1 replication in tissues infected with HIV-1 alone (Figure 6b, light grey line triangle) but not in HIV-1/ HSV-2-infected tissues (Figure $\mathbf{6 b}$, light grey line rectangle). Thus, HSV-2 elevated the concentration of TFV needed to prevent HIV-1 infection. These findings were consistent with our RT-PCR data demonstrating that co-infected tissues treated with TFV at $100 \mu \mathrm{g} \mathrm{ml}^{-1}$ had decreased levels of complete HIV-1 reverse transcripts by sevenfold compared with those treated with TFV at $1 \mu \mathrm{g} \mathrm{ml}^{-1}$ or untreated controls (Figure $6 \mathrm{c}$ ). As levels of HIV-1 reverse transcripts in HIV-1/HSV-2-infected tissues correlated with HIV-1 p24 release, these findings suggest that HSV-2 decreases the anti-HIV-1 activity of TFV by increasing the number of HIV-1-infected cells. Consistent with our previous data (Figure 1a, b and d), we detected enhanced HIV-1 p24 levels (Figure 6a, compare grey line circle and black line diamond) and frequency of HIV-1-infected cells in HIV-1/ HSV-2-infected tissues compared with tissues infected with HIV-1 alone. By evaluating culture supernatants from the same experiment for HSV-2 DNA through day 21, we next addressed whether the lack of anti-HIV-1 activity of TFV at $1 \mu \mathrm{g} \mathrm{ml}^{-1}$ in co-infected tissues was associated with active HSV-2 production. Baseline levels of HSV-2 replication peaked on day 14 and remained the same through day 21 (Figure 6d, black bars), suggesting slow kinetics of HSV-2 replication in ectocervical tissues. Furthermore, we detected similar levels of HSV-2 DNA expression in HIV-1/HSV-2-infected tissues left untreated or treated with TFV at $1 \mu \mathrm{g} \mathrm{ml}^{-1}$ (Figure 6d, compare black bars with grey bars), indicating that at this low concentration TFV was unable to prevent HSV-2 replication within the tissues. In contrast, from days 14-21, levels of HSV-2 DNA were significantly decreased in tissues treated with TFV at $100 \mu \mathrm{g} \mathrm{ml}^{-1}$ (Figure 6d, compare black and white bars). These results demonstrate the development of a productive infection by HSV-2 and suggest that HSV-2 replication has the potential to decrease the anti-HIV-1 activity of low concentrations of TFV in ectocervical tissues. a

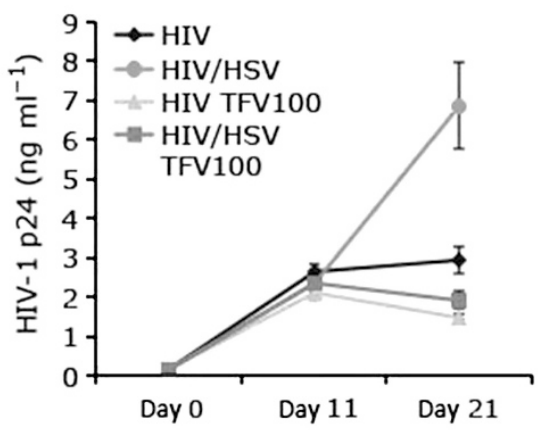

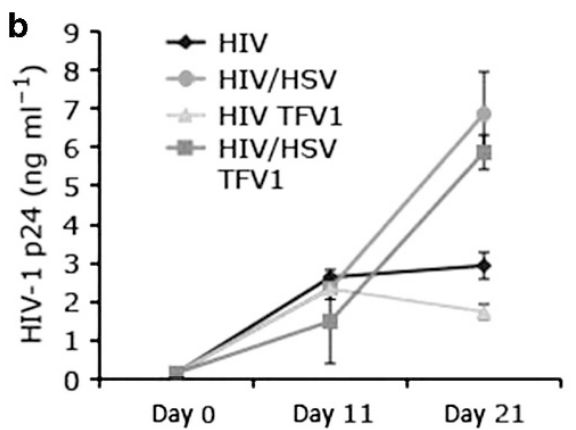
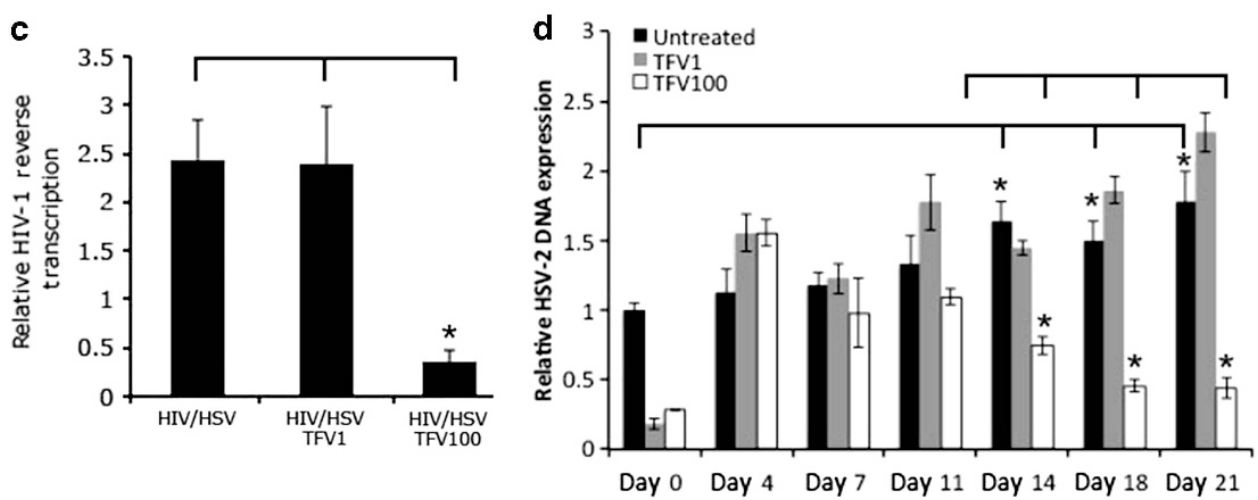

Figure 6 HSV-2 decreases the anti-HIV-1 activity of TFV in ex vivo ectocervical tissues. HIV-1 p24 levels (ng ml ${ }^{-1}$ ) in HIV-1- and HIV-1/HSV-2-infected ectocervical tissues left untreated or treated with TFV at $100 \mu \mathrm{g} \mathrm{ml}^{-1}$ (a) or $1 \mu \mathrm{g} \mathrm{ml}{ }^{-1}$ (b) were measured on days 0,11 , and 21 after infection. ${ }^{*} P<0.05$ for HIV-1/HSV-2-infected tissues left untreated or treated with TFV at $100 \mathrm{~g} \mathrm{ml}^{-1}$, and for untreated HIV-1-infected tissues vs. tissues treated with TFV at either $100 \mu \mathrm{g} \mathrm{ml}^{-1}$ or $1 \mu \mathrm{g} \mathrm{ml}{ }^{-1}$. (c) Levels of complete reverse transcribed HIV-1 DNA in HIV-1/HSV-2-infected tissues left untreated or treated with TFV at $1 \mu \mathrm{g} \mathrm{ml}^{-1}$ and $100 \mu \mathrm{g} \mathrm{ml}^{-1}$ were quantified by RT-PCR on day 21 after infection. All data were normalized to human $\beta$-actin. ${ }^{*} P<0.05$ for HIV-1/HSV-2infected tissues treated with TFV at $100 \mu \mathrm{g} \mathrm{ml}^{-1} \mathrm{vs}$. tissues left untreated or treated with TFV at $1 \mu \mathrm{g} \mathrm{ml} \mathrm{ml}^{-1}$. (d) HSV-2 DNA levels in culture supernatants from HIV-1/HSV-2-infected tissues left untreated (black bars) or treated with TFV at $1 \mu \mathrm{g} \mathrm{ml}^{-1}$ (grey bars) or $100 \mu \mathrm{g} \mathrm{ml}^{-1}$ (white bars) were quantified by RT-PCR through day 21. Results were consistent among three donors and are shown as the mean $\pm s$. d. from three replicates from one representative donor. ${ }^{*} P<0.05$ for HIV-1/HSV-2-infected tissues left untreated or treated with TFV at $100 \mu \mathrm{g} \mathrm{ml}^{-1}$ days 14 through 21 , and for untreated HIV/HSV days 0 through 11 vs. days 14 through 21 after infection. 


\section{DISCUSSION}

Our findings provide novel insights about mechanisms of HSV2 regulation of HIV-1 infection and replication in the genital mucosa. They also describe the effects of co-infection on the anti-HIV efficacy of TFV. HSV-2 enhanced the number of HIV-1-infected cells replicating virus in an ex vivo tissue model of infection. Upregulation of HIV-1 infection and replication by HSV-2 rendered concentrations of TFV that decrease HIV-1 replication in singly infected tissues, ineffective in the context of co-infection. Characterization of the molecular mechanism and cellular pathways underlying the enhanced susceptibility of HIV-1/HSV-2 co-infected ectocervical tissues to HIV-1 infection demonstrated: (1) early upregulation of CD4, CCR5, and CD38 expression and (2) faster kinetics of mucosal CD4 ${ }^{+}$, $\mathrm{CCR}^{+}$, and $\mathrm{CD}_{3} 8^{+}$cell depletion in co-infected tissues compared with tissues infected with HIV-1 alone. Thus, mucosal activation by HSV-2 enhances HIV-1 replication and decreases the anti-HIV-1 activity of TFV.

HSV-2 reactivation results in infiltration of $\mathrm{CD} 4{ }^{+} \mathrm{CCR}^{+} \mathrm{T}$ cells that are susceptible to HIV-1. ${ }^{6,17,19}$ Our findings suggest similar mechanisms of HSV-2 stimulation of HIV-1 replication during acute HSV-2 infection. On day 3, we found faster induction of CD4 RNA expression in dually and HIV-1infected tissues compared with those infected with HSV-2 alone. Similarly, CCR5 RNA expression was upregulated in coinfected tissues compared with those infected with either virus alone, suggesting an accelerated general tissue inflammatory immune response to these viral pathogens. Conversely, on day 5 , CD4 and CCR5 expression was decreased in HIV-1/HSV-2infected tissues, whereas expression remained enhanced in singly infected tissues. HIV-1 downregulated, whereas HSV-2 upregulated CD4 and CCR5 expression on day 7 of infection. As R5-tropic HIV-1 infects CD4 ${ }^{+} \mathrm{CCR}^{+}{ }^{+} \mathrm{T}$ cells in the cervicovaginal mucosa, ${ }^{28}$ our results suggest that earlier kinetics of HIV-1 and HSV-2 induced upregulation of proliferation of, or CD4 and CCR5 expression on, HIV-1 target cells that are subsequently depleted by HIV-1. We speculate that co-infection of cervical tissues by HIV-1 and HSV-2 induces greater levels of pro-inflammatory cytokines, chemokines, and other immune factors, which promote proliferation and activation of immune cells and make them more receptive to HIV-1 infection and replication. This shifts the HIV-1 viral kinetics to earlier time points, leading to an increased CD4 cell death and depletion by day 5 of infection in co-infected tissues compared with that on day 7 in tissues infected with HIV-1 alone. These same factors increase the effective concentration of TFV, and likely any other antiretroviral.

As tissue explant models do not reflect the effect of systemic factors, ${ }^{35}$ this experimental system does not allow for the evaluation of recruitment of inflammatory cell infiltrates. Thus, enhanced CD4 and CCR5 expression likely indicates proliferation of resident HIV-1 target cells induced by HIV-1 and HSV-2. We acknowledge that receptor RNA expression may indicate either immune cell proliferation or enhanced HIV-1 receptor/co-receptor expression on resident mucosal cells. Our assumption of increased $\mathrm{CD} 4{ }^{+}, \mathrm{CCR} 5^{+}$cell proliferation, infection and depletion, however, is consistent with our findings from FACS experiments demonstrating greater frequency of $\mathrm{CD} 4^{+}, \mathrm{CCR} 5^{+}, \mathrm{CD} 38^{+} \mathrm{T}$ cells (FACS data not shown) and increased number of HIV-1 infected cells in co-infected tissues compared to tissues infected with HIV-1 alone (Figure 1b). Our findings are consistent with data in cervicovaginal tissues demonstrating that R5-tropic HIV-1 preferentially infects, replicates and depletes $\mathrm{CD} 4{ }^{+}, \mathrm{CCR}^{+} \mathrm{T}$ cells. ${ }^{28}$ Furthermore, exogenous addition of TFV at inhibitory concentrations for HIV-1 and HSV-2 restored CD4 and CCR5 expression to baseline levels observed in uninfected TFV-treated tissues, and we detected a high frequency of Annexin $\mathrm{V}^{+} \mathrm{CD} 4{ }^{+}$ $\mathrm{T}$ cells in HIV-1/HSV-2- and HIV-1 infected tissues at late stages of HIV- 1 infection suggesting $\mathrm{CD} 4{ }^{+} \mathrm{T}$ cells depletion by HIV-1 (FACS data not shown).

R5-HIV-1 replication in cervicovaginal tissues occurs in $\mathrm{CD} 4{ }^{+} \mathrm{T}$ cells that co-express the activation marker $\mathrm{CD} 38 .{ }^{28}$ Induction of $\mathrm{CD} 38$ expression in bystander $\mathrm{CD} 4{ }^{+} \mathrm{T}$ cells may enhance HIV-1 replication and expand the mucosal pool of HIV-1-infected cells leading to systemic viral dissemination. Our results demonstrating enhanced CD38 expression that waned over time in HIV-1- and HIV-1/HSV-2-infected tissues suggest that either virus is able to induce $\mathrm{CD} 38$ expression on HIV-1 target cells that are subsequently infected and depleted by HIV-1. Modulation of CD38 expression was due to viral replication, as inhibition of HIV-1 and HSV-2 replication by TFV restored CD38 expression to baseline levels. Thus, our results underscore the relevance of $\mathrm{CD} 38$ as a key immune cell activation marker in regulating the susceptibility of mucosal $\mathrm{CD} 4{ }^{+}$target cells to infection by HIV-1 under the setting of co-infection by sexually transmitted pathogens.

Epidemiological data support a synergistic interaction between HIV-1 and HSV-2. ${ }^{1,4,15}$ HIV-1 and HSV-2 infections facilitate each other's acquisition, transmission, and disease progression. ${ }^{25,36}$ Our findings of enhanced HIV-1 and HSV-2 replication in co-infected ectocervical tissues represent critical experimental evidence of this synergistic interaction. Early studies suggest that HIV-1 and HSV-2 can co-infect lymphocytes in vitro and in vivo. ${ }^{37}$ Several HSV-2 regulatory proteins seem to upregulate HIV-1 replication by interacting with the HIV long-terminal repeat. ${ }^{37}$ In this study, the specific issue of HIV-1/HSV-2 co-infection within the same cell and thus a direct mechanism of HIV-1 upregulation by HSV-2 was not addressed. However, the facts that HSV-2 mainly infects skin and mucosal epithelial cells, ${ }^{38}$ and HIV-1 primarily infects intraepithelial or sub-mucosal CD4 ${ }^{+} \mathrm{T}$ cells ${ }^{28,39}$ suggest that the mechanism(s) of HSV-2-mediated upregulation of HIV-1 infection is indirect. Similarly, HSV-2 infection of epithelial cells results in the production of chemokines, cytokines, or additional immune factors that activate or induce resident HIV-1 target cells to proliferate. Upregulation of one of these immune factors CXCL9 has been reported following HSV-2 infection of genital epithelial cells. ${ }^{39}$ Furthermore, CXCL9 expression in supernatants from HSV-2-infected epithelial cells induced the migration of $\mathrm{CD} 4{ }^{+} \mathrm{T}$ cells in vitro compared with supernatants from uninfected control cells. ${ }^{39}$ Experiments are 
underway to address whether CXCL9 induction by HSV-2 has a role in enhancing HIV-1 target cell activation, proliferation, and thus HIV-1 replication in ectocervical tissues.

TFV is the only anti-HIV-1 microbicide with proven efficacy in macaques and human studies. ${ }^{26,40,41}$ In the landmark trial CAPRISA 004, TFV $1 \%$ gel demonstrated a $39 \%$ reduction in HIV-1 acquisition by women compared with placebo. ${ }^{26}$ TFV effectiveness increased to $54 \%$ in women who self-reported greater than $80 \%$ adherence to the protocol. We postulate that in addition to poor adherence, ${ }^{42}$ partial protection by TFV gel may have been due to cervicovaginal microenvironmental factors that enhanced mucosal susceptibility to HIV-1. We found that TFV at low concentrations $\left(1 \mu \mathrm{g} \mathrm{ml}^{-1}\right)$ decreased HIV-1 replication in HIV-1-infected tissues but not in HIV-1/ HSV-2-co-infected tissues. As HSV-2 enhanced HIV-1 target cell activation, viral infection, and replication, our findings suggest immune cell activation as the mechanisms by which HSV-2 decreased the anti-HIV-1 efficacy of TFV. The lack of efficacy of TFV at low concentrations was likely related to productive HSV-2 replication, as TFV at concentrations ranging from $50 \mu \mathrm{g} \mathrm{ml}^{-1}$ to $100 \mu \mathrm{g} \mathrm{ml}^{-1}$ that prevented HSV-2 infection in ectocervical tissues was very effective in downregulating HIV-1 replication in HIV-1- and HIV-1/HSV-2infected tissues. Remarkably, CAPRISA participants had HSV-2 infection rates greater than $50 \%$, and in this study HSV-2 infection was associated with HIV-1 acquisition. ${ }^{26,43}$

Further characterization of the mechanism(s) of decreased anti-HIV-1 efficacy of TFV suggests that greater numbers of HIV-1-infected cells and levels of viral replication in dually infected tissues are the main contributors. A two- to threefold increase in levels of tenofovir-diphospate (dp), the active metabolite, has been reported in nonactivated compared with activated peripheral blood mononuclear cells (PBMC); ${ }^{44,45}$ hence, immune cell activation by HSV-2 may compromise TFV efficacy by decreasing its intracellular phosphorylation. By increasing proliferation, immune cell activation may have reduced intracellular levels of TFV-dp. As TFV-dp is a $2^{\prime}$ deoxyadenosyne triphosphate (dATP) analog that competes with endogenous dATP for incorporation into the growing HIV DNA chain, it is also possible that immune cell activation by HSV-2 may increase the concentration of endogenous dATP and significantly lower the TFV-dp/dATP ratio in activated cells, resulting in decreased antiviral activity. ${ }^{46}$

Anti-HIV-1 and HSV-2 activity of TFV has been previously described. ${ }^{26,33,34,47}$ We observed that TFV at 100 and $50 \mu \mathrm{g} \mathrm{ml}^{-1}$ suppressed replication of both viruses, whereas TFV at $1 \mu \mathrm{g} \mathrm{ml}^{-1}$ only suppressed HIV-1. Our findings are consistent with the concentration range that inhibited HIV-1 and HSV-2 in lymphoid and cervicovaginal tissues ex vivo, ${ }^{33}$ and with the anti-HIV-1 and anti-HSV-2 activity achieved intravaginally with a topical application of TFV $1 \%$ gel. $^{26}$

HSV-2 replication is characterized by near constant viral shedding rather than the latent phase between episodes of symptomatic ulcer disease. ${ }^{36}$ Our finding of low levels of HSV -2 replication in ectocervical tissues treated with TFV at $1 \mu \mathrm{g} \mathrm{ml}^{-1}$ suggests that women exposed to TFV while actively replicating HSV-2 will be at significant higher risk for acquiring HIV-1, if TFV does not prevent HSV-2 infection and replication in the genital mucosa. It would be important to achieve higher concentrations of TFV and TFV-dp in cervicovaginal tissues, as those possibly achieved by new TFV prodrugs and delivery systems. ${ }^{48,49}$

Taken together, our data show increased HIV-1 replication in cervicovaginal tissues co-infected with HSV-2, which was associated with early increase in CD4, CCR5, and CD38 tissue expression and faster decline possibly due to enhanced cell death and depletion of HIV-1-infected cells. Our data also show that co-infected tissues required higher concentration of TFV to block HIV-1 infection and replication. Therefore, in addition to adherence to microbicide use, biological HIV-1 risk factors such as co-infection by HSV-2 must be considered in order to achieve higher levels of mucosal protection against HIV-1. These susceptibility enhancing factors should be taken into account when planning and interpreting data from clinical effectiveness trials. Blocking sexually transmitted pathogens, and HSV-2 in particular, may further improve the success of anti-HIV-1 microbicides and other HIV prevention strategies.

\section{METHODS}

Tissue samples. Ectocervical tissues were obtained from HIV-1 and HSV-2 seronegative women who were undergoing routine hysterectomy at Dartmouth-Hitchcock Medical Center (DHMC) for benign medical conditions including prolapse and uterine fibroids. All tissues were obtained according to an Institutional Review-Board-approved protocol.

For each experimental condition, 3-6 nonpolarized tissue blocks were placed in individual wells of 48-well plates and infected with HSV-2 or HIV-1 alone or in combination within 3-4h of surgery. Under our culture conditions, tissue explants can be maintained for up to 18-21 days without a significant decrease in viability, as determined by lactate dehydrogenase assay (Cytotoxicity Detection Kit, Roche, Indianapolis, IN (data not shown)).

Viral infection. Tissue blocks were infected with $4 \times 10^{4}$ p.f.u. per $\mathrm{ml}$ of HSV -2 clinical isolate 186 , before infection with $1 \times 10^{4} \mathrm{TCID}_{50}$ of cellfree R5-tropic HIV-1 $1_{\mathrm{BaL}}$ (NIH AIDS Research \& Reference Reagent Program, Bethesda, MD). After overnight incubation at $37^{\circ} \mathrm{C}$, tissues were washed to remove residual input virus and cultured for up to 21 days. A sample of the culture supernatants was collected after the final wash (day 0 ), and again on days $4,7,11,14,18$, and 21 after infection and evaluated for p24 antigen by ELISA (Perkin Elmer, Boston, MA). At each time point, one-half of the culture supernatant was removed and replenished with an equivalent volume of fresh media. When indicated, TFV at different concentrations was added only once for $6 \mathrm{~h}$ before HIV-1 infection. The HIV-1 stock was generated in human PBMC and titered in cultures of TZM-bl target cells (NIH AIDS Research and Reference Reagent Program). HSV-2 stocks were generated and titered in Vero cells (ATCC, Catalog \# CCL-81).

Nucleic acid isolation. Tissue blocks were homogenized with a scintered glass homogenizer (Kontes, Vineland, NJ) and the lysate applied to a Shredder column (Qiagen, Valencia, CA). The supernatant was subjected to RNA isolation using the RNeasy-Plus kit (Qiagen). Genomic DNA was extracted by using the QIAmp DNA mini kit (Qiagen) on days 11 and 21 after infection. Day 11 is one of the earliest time points where we consistently detected HIV-1 DNA expression, and day 21 is the day where experiments were terminated. 
HSV-2 replication. HSV-2 DNA was evaluated by a quantitative RT-PCR amplification of tissue culture supernatants collected through day 21 using SYBR-green (Applied Biosystems, Warrington, UK). Supernatants $(10 \mu \mathrm{l})$ were amplified using the forward primer $5^{\prime}$-CGACGGCGATGCGCCCCAGC- $3^{\prime}$ and the reverse primer $5^{\prime}$-CGCATCAAGACCACCTCCTC-3' targeting glyprotein B of isolate 186 to yield a 99-base pair (bp) product.

HIV-1 reverse transcription and integration. HIV-1 DNA was detected by a two-step RT-PCR amplification of genomic DNA (250 ng) using the SK431 forward primer 5'-TGCTATGTCAG TTCCCCTTGGTTCTCT- $3^{\prime}$ and the GAG1 reverse primer $5^{\prime}$-TCA GCCCAGAAGTAATACCCATGT-3'. Five microliters of this PCR product was used in a second-round amplification using GAG1 and the GAG2 reverse primer $5^{\prime}$-CACTGTGTTTAGCATGGTGTTT- $3^{\prime}$ to yield a 81-bp product. These primers detect nearly completely synthesized viral DNA.

HIV-1 integration was detected by a two-step RT-PCR assay where the first-round PCR amplifies DNA sequence between the HIV-1 proviral sequence and the nearest chromosomal ALU elements, and the nested PCR amplifies HIV-1 products pre-amplified in the first round. The first-round amplification was performed using forward and reverse primers specific for the ALU elements: 5'-TCCCAGC TACTGGGGAGGCTGAGG- $3^{\prime}$ and $5^{\prime}$-GCCTCCCAAAGTGCTGG GATTACAG-3', respectively and the HIV-1 specific primer: $5^{\prime}$ ATGCCACGTAAGCGAAACTCTGGCTAACTAGGGAACCCACT G-3' extended at its $5^{\prime}$ end with a lambda phage heel sequence. Five microliters of this product was used in a second-round amplification, using primers targeting the heel-specific sequence $5^{\prime}$-ATGCCACGTAAGCGAAACT- $3^{\prime}$ and the HIV-1 gag sequence $5^{\prime}$ CCTGCGTCGAGAGAGCTCCTCTGG-3' to yield a 159-bp fragment. ${ }^{50}$ Values of reverse transcribed and integrated HIV-1 DNA were normalized to endogenous human $\beta$-actin amplified with the forward primer $5^{\prime}$-CACTCTTCCAGCCTTCCTTCC- $3^{\prime}$ and the reverse primer $5^{\prime}$-CTGTGTTGGCGTACAGGTCT- $3^{\prime},{ }^{50}$ and expressed relative to HIV-1 infected tissues on day 11 (set to 1 ).

Gene transcription. Five micrograms of total RNA was reverse transcribed using Superscript III reverse transcriptase (Invitrogen, Carlsbad, CA, USA) and subjected to RT-PCR using the forward primer $5^{\prime}$-GGGCGGCGACTGAATTGGGT- $3^{\prime}$ and the reverse primer $5^{\prime}$-CCGCTTCTTCCTGCCATAGGAGATG-3' complementary to the genome of $\mathrm{HIV}-1_{\mathrm{BaL}}$ to yield a 160 -bp product from spliced HIV-1 transcripts. CD4 transcription was evaluated using the forward primer $5^{\prime}$-GGCAGTGTCTGCTGAGTGA- $3^{\prime}$ and the reverse primer $5^{\prime}$-GTGGGCAGAACCTTGATGTTG- $3^{\prime}$ that yield a 64 -bp product from spliced CD4 transcripts. CCR5 was evaluated using the forward primer $5^{\prime}$-GCTGTCGTCCATGCTGTGTT- $3^{\prime}$ and the reverse primer $5^{\prime}$-CAGCCACCACCCAAGTGAT- $3^{\prime}$ that yield a 85 -bp product. CD38 transcription was detected using the forward primer $5^{\prime}$-GGAGAGCCCAACTCTGTCT- $3^{\prime}$ and the reverse primer $5^{\prime}$-CGA GCACCACGACGAGGAT- $3^{\prime}$ that yield a 61 -bp product. HIV-1, CD4, CCR5, and CD38 transcription values were normalized to endogenous human GAPDH amplified with the forward primer $5^{\prime}$-TC CCATCACCATCTTCCAG- $3^{\prime}$ and the reverse primer $5^{\prime}$-GACTCC ACGACGTACTCA-3'.

Statistical analysis. Analysis of data sets was performed by two-tailed, unpaired Student's $t$-test using Prism 5 (Graph Pad software, Inc.). $P$-values of $<0.05$ were considered significant.

\section{ACKNOWLEDGMENTS}

This work was supported by the Department of Veterans Affairs, Merit Review Program (SNA), and a pre-clinical award (PPA-10-084) from CONRAD (SNA). We thank Dr D Koelle, University of Washington, Seattle, WA, for kindly providing us with the HSV-2 clinical isolate 186 . We thank the Department of Medicine, Section of Anatomic Pathology, DHMC, Lebanon, $\mathrm{NH}$, USA, for their assistance in procuring ectocervical tissues.

\section{DISCLOSURE}

The authors declare no conflict of interest.

\section{(c) 2014 Society for Mucosal Immunology}

\section{REFERENCES}

1. Ghebremichael, M., Habtzgi, D. \& Paintsil, E. Deciphering the epidemic synergy of herpes simplex virus type 2 (HSV-2) on human immunodeficiency virus type 1 (HIV-1) infection among women in sub-Saharan Africa. BMC Res. Notes 5, 451 (2012).

2. Mmbaga, E.J., Leyna, G.H., Stray-Pedersen, B. \& Klepp, K.I. Herpes simplex virus type-2 and human immunodeficiency virus infections in a rural population in Kilimanjaro Tanzania. East Afr. J. Public Health 8, 28-32 (2011).

3. Venkatesh, K.K. et al. The relative contribution of viral and bacterial sexually transmitted infections on HIV acquisition in southern African women in the Methods for Improving Reproductive Health in Africa study. Int. J. STD AIDS 22, 218-224 (2011).

4. Wald, A. \& Link, K. Risk of human immunodeficiency virus infection in herpes simplex virus type 2-seropositive persons: a meta-analysis. J. Infect. Dis. 185, 45-52 (2002).

5. Horbul, J.E., Schmechel, S.C., Miller, B.R., Rice, S.A. \& Southern, P.J. Herpes simplex virus-induced epithelial damage and susceptibility to human immunodeficiency virus type 1 infection in human cervical organ culture. PLOS ONE 6, e22638 (2011).

6. Zhu, J. et al. Persistence of HIV receptor-positive cells after HSV-2 reactivation is a potential mechanism for increased HIV acquisition. Nat. Med. 15, 886-892 (2009).

7. Drannik, A.G., Nag, K., Sallenave, J.M. \& Rosenthal, K.L. Antiviral activity of trappin-2 and elafin in vitro and in vivo against genital herpes. J. Virol. 87, 7526-7538 (2013).

8. Fakioglu, E. et al. Herpes simplex virus downregulates secretory leukocyte protease inhibitor: a novel immune evasion. J. Virol. 82, 9337-9344 (2008).

9. Sheth, P.M. et al. Coinfection with herpes simplex virus type 2 is associated with reduced HIV-specific $T$ cell responses and systemic immune activation. J. Infect. Dis. 197, 1394-1401 (2008).

10. Li, H. et al. HSV-2 induces TLRs and NF-kappaB-dependent cytokines in cervical epithelial cells. Biochem. Biophys. Res. Commun. 379, 686-690 (2009).

11. Goodkin, M.L., Ting, A.T. \& Blaho, J.A. NF-kappa B is required for apoptosis prevention during herpes simplex virus type 1 infection. J. Virol. 77, 7261-7280 (2003)

12. Asin, S.N., Eszterhas, S.K., Rollenhagen, C., Heimberg, A.M. \& Howell, A. HIV type 1 infection in women: increased transcription of HIV type 1 in ectocervical tissue explants. J. Infect. Dis. 200, 965-972 (2009).

13. Rollenhagen, C. \& Asin, S.N. IL-8 decreases HIV-1 transmission in peripheral blood lymphocytes and ectocervical tissue explants. J. Acquir. Immune Defic. Syndr. 54, 463-469 (2010).

14. Rollenhagen, C. \& Asin, S.N. Enhanced HIV-1 replication in ex vivo ectocervical tissues from post-menopausal women correlates with increased inflammatory responses. Mucosal Immunol. 4, 671-681 (2011).

15. Krzyzowska, M. et al. HSV-2 regulates monocyte inflammatory response via the Fas/FasL pathway. PLoS One 8, e70308 (2013).

16. lijima, N., Mattei, L.M. \& Iwasaki, A. Recruited inflammatory monocytes stimulate antiviral Th1 immunity in infected tissue. Proc. Natl. Acad. Sci. USA 108, 284-289 (2011).

17. Nakanishi, Y., Lu, B., Gerard, C. \& Iwasaki, A. CD8 (+) T Iymphocyte mobilization to virus-infected tissue requires CD4 $(+)$ T-cell help. Nature 462, 510-513 (2009).

18. Lund, J.M., Linehan, M.M., lijima, N. \& Iwasaki, A. Cutting edge: plasmacytoid dendritic cells provide innate immune protection against mucosal viral infection in situ. J. Immunol. 177, 7510-7514 (2006).

19. Koelle, D.M., Schomogyi, M. \& Corey, L. Antigen-specific Tcells localize to the uterine cervix in women with genital herpes simplex virus type 2 infection. J. Infect. Dis. 182, 662-670 (2000).

20. Biancotto, A. et al. HIV-1 induced activation of CD4 + T cells creates new targets for HIV-1 infection in human lymphoid tissue ex vivo. Blood 111, 699-704 (2008). 
21. McKinnon, L.R. \& Kaul, R. Quality and quantity: mucosal CD4 + Tcells and HIV susceptibility. Curr. Opin. HIV AIDS 7, 195-202 (2012).

22. Jaspan, H.B. et al. Immune activation in the female genital tract during HIV infection predicts mucosal CD4 depletion and HIV shedding. J. Infect. Dis. 204, 1550-1556 (2011).

23. Shao, $Y$. et al. Keratinocytes play a role in the immunity to Herpes simplex virus type 2 infection. Acta Virol. 54, 261-267 (2010).

24. Carr, D.J. \& Tomanek, L. Herpes simplex virus and the chemokines that mediate the inflammation. Curr. Top. Microbiol. Immunol. 30, 47-65 (2006).

25. Thurman, A.R. \& Doncel, G.F. Herpes simplex virus and HIV: genital infection synergy and novel approaches to dual prevention. Int. J. STD AIDS 23, 613-619 (2012).

26. Abdool Karim, Q. et al. Effectiveness and safety of tenofovir gel, an antiretroviral microbicide, for the prevention of HIV infection in women. Science 333, 1168-1174 (2010).

27. Josefsson, L. et al. Single cell analysis of lymph node tissues from HIV-1 infected patients reveals that the majority of CD4 + T-cells contain one HIV-1 DNA molecule. PLoS Pathog. 9, e1003432 (2013).

28. Saba, E. et al. HIV-1 sexual transmission: early events of HIV-1 infection of human cervico-vaginal tissue in an optimized ex vivo model. Mucosal Immunol. 3, 280-290 (2010).

29. Gorry, P.R. \& Ancuta, P. Coreceptors and HIV pathogenesis. Curr. HIVIAIDS Rep. 8, 45-53 (2011).

30. Grivel, J.C., Shattock, R.J. \& Margolis, L.B. Selective transmission of R5 HIV-1 variants: where is the gatekeeper? J. Transl. Med. 9 (Suppl 1), S6 (2011).

31. Grivel, J.C. et al. HIV-1 pathogenesis differs in rectosigmoid and tonsillar tissues infected ex vivo with CCR5- and CXCR4-tropic HIV-1. AIDS 21 1263-1272 (2007).

32. Margolis, L. \& Shattock, R. Selective transmission of CCR5-utilizing HIV-1: the "gatekeeper" problem resolved?. Nat. Rev. Microbiol. 4, 312-317 (2006).

33. Andrei, G. et al. Topical tenofovir, a microbicide effective against HIV, inhibits herpes simplex virus-2 replication. Cell Host Microbe 10, 379-389 (2011).

34. Vibholm, L. et al. Antiviral and immunological effects of tenofovir microbicide in vaginal herpes simplex virus 2 infection. AIDS Res. Hum. Retroviruses 28, 1404-1411 (2012).

35. Grivel, J.C. \& Margolis, L. Use of human tissue explants to study human infectious agents. Nat. Protoc. 4, 256-269 (2009).

36. Barnabas, R.V. \& Celum, C. Infectious co-factors in HIV-1 transmission herpes simplex virus type-2 and HIV-1: new insights and interventions. Curr. HIV Res. 10, 228-237 (2012).

37. Palù, G., Benetti, L. \& Calistri, A. Molecular basis of the interactions between herpes simplex viruses and HIV-1. Herpes 8, 50-55 (2001).
38. Cunningham, A.L. et al. The cycle of human herpes simplex virus infection: virus transport and immune control. J. Infect. Dis. 194 (Suppl 1), S11-S18 (2006).

39. Huang, W. et al. Herpes simplex virus type 2 infection of human epithelial cells induces CXCL9 expression and CD4 + T cell migration via activation of p38-CCAAT/enhancer-binding protein- $\beta$ pathway. J. Immunol. 188, 6247-6257 (2012).

40. Pham, P.A. \& Gallant, J.E. Tenofovir disoproxil fumarate for the treatment of HIV infection. Expert Opin. Drug Metab. Toxicol. 2, 459-469 (2006).

41. Dobard, C. et al. Durable protection from vaginal simian-human immunodeficiency virus infection in macaques by tenofovir gel and its relationship to drug levels in tissue. J. Virol. 86, 718-725 (2012).

42. Grobler, A. \& Abdool Karim, S. Declining adherence is a more likely explanation than frailty of the apparent decline in efficacy in the CAPRISA 004 trial. AIDS 26, 2261 (2012). author reply 2262-3.

43. Naranbhai, V. et al. Innate immune activation enhances HIV acquisition in women, diminishing the effectiveness of tenofovir microbicide gel. J. Infect. Dis. 206, 993-1001 (2012).

44. Robbins, B.L., Wilcox, C.K., Fridland, A. \& Rodman, J.H. Metabolism of tenofovir and didanosine in quiescent or stimulated human peripheral blood mononuclear cells. Pharmacotherapy 23, 695-701 (2003).

45. Balzarini, J., Van Herrewege, Y. \& Vanham, G. Metabolic activation of nucleoside and nucleotide reverse transcriptase inhibitors in dendritic and Langerhans cells. AIDS 16, 2159-2163 (2002).

46. Goicoechea, M. et al. Abacavir and tenofovir disoproxil fumarate co-administration results in a nonadditive antiviral effect in HIV-1-infected patients. AIDS 24, 707-716 (2010).

47. Ballzarini, J. et al. A Multi-targeted drug candidate with dual anti-HIV and anti-HSV activity. PloS Pathog. 9, e1003456 (2013).

48. Johnson, T.J. et al. A 90-day tenofovir reservoir intravaginal ring for mucosal HIV prophylaxis. Antimicrob. Agents Chemother. 56, 6272-6283 (2012).

49. Smith, J.M. et al. Intravaginal ring eluting tenofovir disoproxil fumarate completely protects macaques from multiple vaginal simian-HIV challenges. Proc. Natl. Acad. Sci. USA 110, 16145-16150 (2013).

50. Asin, S.N., Heimberg, A.M., Eszterhas, S.K., Rollenhagen, C. \& Howell, A.L. Estradiol and progesterone regulate HIV type 1 replication in peripheral blood cells. AIDS Res. Hum. Retroviruses 24, 701-716 (2008).

(c) (1) $(\Theta)$ This work is licensed under the Creative Commons Attribution-NonCommercial-No Derivative Works 3.0 Unported License. To view a copy of this license, visit http://creativecommons.org/licenses/by-nc-nd/3.0/ 